\title{
Integrated Analysis of the m6A-Related IncRNA Identified IncRNA ABALON/miR-|39-3p/NOB I Axis Was Involved in the Occurrence of Lung Cancer
}

\author{
Xinhong $\operatorname{Liu}^{\mathrm{I}-4, *}$ \\ Xin Zuo ${ }^{5, *}$ \\ Lijun $\mathrm{Ma}^{\mathrm{I}-4}$ \\ Qin Wang ${ }^{\mathrm{I}-4}$ \\ Lilan Zhu ${ }^{\mathrm{I}-4}$ \\ $\mathrm{Li} \mathrm{Li}^{\mathrm{l}-4}$ \\ Xin Zhao (iD ${ }^{2-4}$ \\ 'College of Biological and Chemical \\ Engineering, Chongqing University of \\ Education, Chongqing, People's Republic \\ of China; ${ }^{2}$ Chongqing Collaborative \\ Innovation Center for Functional Food, \\ Chongqing University of Education, \\ Chongqing, People's Republic of China; \\ ${ }^{3}$ Chongqing Engineering Research Center \\ of Functional Food, Chongqing University \\ of Education, Chongqing, People's \\ Republic of China; ${ }^{4}$ Chongqing \\ Engineering Laboratory for Research and \\ Development of Functional Food, \\ Chongqing University of Education, \\ Chongqing, People's Republic of China; \\ ${ }^{5}$ Department of Gastroenterology, The \\ Sixth People's Hospital of Chongqing, \\ Chongqing, People's Republic of China
}

*These authors contributed equally to this work
Correspondence: Xin Zhao

Chongqing Collaborative Innovation Center for Functional Food, Chongqing University of Education, Xuefu Main Street 9, Chongqing, 400067, People's Republic of China

Tel +86-23-62653650

Email zhaoxin@cque.edu.cn
Background: Lung cancer has the characteristics of early metastasis, high recurrence, and high mortality rate despite emerging advances in diagnostic. Early diagnosis can significantly improve the patient's chances of cure and survival.

Purpose: This study aimed to identify and assess a prognostic lncRNA/miRNA/gene signature in patients with lung cancer.

Methods: Pearson correlation analysis, univariate Cox analysis and LASSO Cox analysis were used to construct a lung cancer prognostic risk model based on m6A-related lncRNA. The interaction between IncRNA-miRNA-gene was verified by luciferase reporter gene experiment. Results: The Pearson correlation analysis determined that 1655 lncRNAs significantly correlated with the expression of m6A genes. A lung cancer prognostic risk model, including 14 m6A-related lncRNAs, was constructed through univariate Cox analysis and least absolute shrinkage and selection operator (LASSO) Cox analysis. ABALON was identified as the key lncRNA through cluster analysis and gene expression difference analysis.

Conclusion: It was experimentally verified that ABALON acted as a competing endogenous RNA by sponging miR-139-3p and indirectly regulated the expression of NOB1. This study provided a new biological target for the early diagnosis of lung cancer and a new direction for studying the mechanism of lung cancer.

Keywords: lncRNA, lung cancer, miRNA, N6-methyladenosine, prognostic biomarker

\section{Introduction}

Lung cancer remains the leading cause of cancer-related death worldwide, and the high incidence rates are worrisome. ${ }^{1}$ The five-year survival rate for lung cancer is only $18 \%$, and early diagnosis can significantly improve the patient's chances of cure and survival. ${ }^{2}$ Although many advances have been made in the diagnosis and treatment of lung cancer in recent years, the understanding of lung cancer prognostic molecular markers is still lacking. ${ }^{3}$ Epigenetic modifiers, including long coding RNAs (lncRNAs) and microRNAs (miRNAs), have important roles in the development and progression of lung cancer. ${ }^{4,5}$ Thus, it is particularly important to further explore new lncRNAs and miRNAs as diagnostic targets of lung cancer and clarify their molecular mechanisms.

LncRNAs are noncoding transcripts usually longer than $200 \mathrm{nt}$ that have recently emerged as one of the largest and significantly diverse RNA families and 
can modulate various biological functions at epigenetic, transcriptional, and posttranscriptional levels, or directly regulate protein activity. ${ }^{6,7}$ MiRNAs are small RNAs that regulate the expression of complementary messenger RNAs (mRNAs) and can regulate tumor progression and metastasis by interacting with target genes. ${ }^{8,9}$ Studies have shown that IncRNAs act as competing endogenous RNA (ceRNA) by sponging miRNAs and indirectly regulate gene expression and then participate in the occurrence of cancer, including lung cancer. ${ }^{10-14}$ N6-methyladenosine (m6A) modification, the most abundant epigenetic methylated modification of mRNAs and noncoding RNAs (ncRNAs), is vital in RNA splicing, export, stability, and translation. ${ }^{15,16}$ Numerous studies show that m6A mediates the occurrence and development of lung cancer by regulating biological processes such as glycolysis and cell cycle. ${ }^{17-19}$ Furthermore, certain m6A regulatory proteins responsible for abnormal m6A modifications on ncRNAs are also involved in cancer cell proliferation, invasion, and drug resistance, suggesting a potential association between cancer and m6A ncRNA modification, and thus, offering a new opportunity for cancer diagnosis and treatment. ${ }^{20,21}$

Based on The Cancer Genome Atlas (TCGA) dataset ( $\mathrm{n}=$ 1145), the prognostic significance of m6A-related lncRNAs was identified by bioinformatics and statistical analysis of data from lung cancer tissue samples and adjacent nontumor tissue samples. This study showed that 22 m6A-related lncRNAs were significantly related to the survival of lung cancer. Also, a prognostic risk model for lung cancer was constructed through LASSO Cox analysis. After a series of differential analysis of prognostic-related m6A-related IncRNAs, finally, ABALON was determined as the key IncRNA. Using the miRDB, miRTarBase, and TargetScan websites, it was predicted that miR-139-3p could target ABALON and NOB1. Finally, it was experimentally verified that ABALON acted as a ceRNA by sponging miR-139-3p and indirectly regulated the expression of $N O B 1$.

This study showed that ABALON could be used as a new marker for the diagnosis of lung cancer and verified the possible molecular mechanism of ABALON in the occurrence of lung cancer.

\section{Materials and Methods}

\section{Acquisition and Re-Annotation of Public Data}

The gene expression quantification data and the corresponding clinical information of patients with lung cancer were obtained from TCGA data portal (https://tcga-data. nci.nih.gov/tcga/). The obtained population included 1037 lung cancer tissue samples and 108 adjacent nontumor tissue samples. After obtaining the raw lung cancer data from TCGA database, the data were re-annotated using the gene transfer format (ftp://ftp.ensembl.org/pub/release-89/ gtf/homo sapiens/), and the RNA properties (mRNA and IncRNA) were recorded.

\section{Co-Expression Analysis of m6A-Related Genes and IncRNA}

According to previous studies, 23 m6A-related genes (METTL3, METTL14, METTL16, WTAP, VIRMA, ZC3H13, RBM15, RBM15B, YTHDC1, YTHDC2, YTHDF1, YTHDF2, YTHDF3, HNRNPC, FMR1, LRPPRC, HNRNPA2B1, IGFBP1, IGFBP2, IGFBP3, $R B M X, F T O$, and $A L K B H 5)$ were compiled, ${ }^{2-25}$ and the expression data of these 23 genes in 514 samples were extracted. Subsequently, the co-expression analysis of m6A-related genes and lncRNAs was performed to determine the m6A-related IncRNAs, and a co-expression network of m6A-related genes and lncRNAs was constructed. The correlation between the m6A-related genes and IncRNAs was calculated using Pearson's method. LncRNAs with an absolute value of correlation coefficients $>0.4$ and $P$ values $<0.001$ were considered immunerelated lncRNAs.

\section{Identification of Prognostic-Related m6A-Related IncRNAs}

The survival status and survival time data of lung cancer samples were extracted and merged with the expression data of m6A-related IncRNAs, the clinical information is shown in Supplementary Table 1 . The "survival" package of the R software was used to compare all lncRNAs with the survival time and survival status to determine the prognostic-related IncRNAs. Immediately, the K-means algorithm was used to analyze the survival of these lncRNAs in patients with lung cancer to determine more credible survival-related lncRNAs. A $P$ value $\leq 0.01$ was considered statistically significant.

\section{Construction of the Prognostic Model}

The LASSO method was used to select key prognosticand m6A-related lncRNAs from the lncRNAs found to be significant in the univariate Cox analysis. Most potential indicators were shrunk to zero, and a relatively small 
number of indicators remained with weights of nonzero. Finally, the key prognostic- and m6A-related IncRNAs were used to construct a prognostic model and generate a forest map. The risk score of each patient with lung cancer was calculated as follows:

$$
\text { Risk score }=\sum_{\mathrm{i}=1}^{\mathrm{n}} \operatorname{Coef}_{\mathrm{i}}{ }^{*} \mathrm{x}_{\mathrm{i}}
$$

where $\operatorname{Coef}_{i}$ means the coefficients and $x_{i}$ is the fragments per kilobase of transcript per million mapped reads normalized expression value of each m6A-related lncRNAs.

\section{Assessment of the Prognostic Model}

All patients with lung cancer were randomly divided into two groups: the train and test groups. Then, these two groups were further divided into high- and low-risk groups using the median value of the risk value as the dividing line. The Kaplan-Meier survival curves between the train and test groups were analyzed and drawn using the "survival" and "survminer" package of the R software. The time-dependent receiver operating characteristic (ROC) curve analysis $(1,3$, and 5 years) was performed to estimate the survival predictive accuracy of the prognostic model. The performance of the prognostic model was evaluated by assessing the area under the ROC (AUC) curve. The risk scores were calculated based on the formula generated through the LASSO Cox regression model. Patient's age, sex, cancer stage, and other data were preprocessed and then integrated with the risk value. Then, single- and multifactor independent prognostic analyses were performed using the "survival" package of the $\mathrm{R}$ software.

\section{Cluster and Gene Set Enrichment Analysis of IncRNAs}

All samples were clustered based on the expression levels of lncRNAs, which significantly related to lung cancer survival in univariate Cox analysis, and a survival analysis was conducted for different clusters by "ConsensusClusterPlus," "survival," and "survminer" package of the R software. For investigating the potential biological process and pathway involved in the molecular heterogeneity between the different clusters, the gene set enrichment analysis (GSEA) software was used on different clusters. A $P$ value $\leq 0.05$ was considered statistically significant.

\section{Immune Infiltration and Tumor Microenvironment Analysis}

Configuration files of gene expression in 22 immune cells were obtained using the CIBERSORT software, which were combined with the gene expression matrix of lung cancer samples to obtain the content of 22 immune cells in each sample. Then, the difference analysis of immune cells was carried out between different clusters. ESTIMATE was used to perform matrix scoring and immune scoring on all lung cancer samples, and the differences were analyzed in stromal cell and immune cell content between different clusters. The "LIMMA" package of the $\mathrm{R}$ software was used to analyze whether a correlation existed between the 22 immune cells and the risk score of patients with lung cancer. A $P$-value $\leq 0.05$ was considered statistically significant.

\section{Model IncRNA Differential Expression Analysis}

The "ggplot2" and "ggpubr" package of the R software was used to analyze the differences in the expression of model lncRNAs between different cancer and normal samples, different clusters, and high- and low-risk groups to further confirm which IncRNAs have a key role in the model and provide a clearer direction for follow-up research. A $P$-value $\leq 0.05$ was considered statistically significant.

\section{Subcellular Localization and Target Gene Prediction}

First, the lncLocator online database (http://www.csbio. sjtu.edu.cn/bioinf/lncLocator/) was used for subcellular localization of the key lncRNA, and the target miRNA was predicted through miRDB (http://mirdb.org/custom.

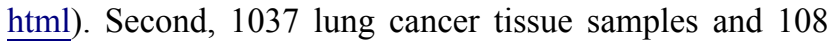
adjacent nontumor tissue samples were obtained through differential analysis of miRNA expression data to find miRNAs that were differentially expressed between patients with lung cancer and normal samples. |log2 fold change $\mid>2$ and $P<0.05$ were considered statistically significant. The miRNAs that were highly expressed in normal lung cancer samples and bound to key lncRNAs were selected as key miRNAs. Finally, three online databases miRDB, miRTarBase (http://mirtarbase.cuhk.edu.cn/php/ index.php), and TargetScan (http://www.targetscan.org/) were used to predict the target genes of key miRNAs. The genes predicted by all three websites were identified 
as candidate target genes. The Gene Expression Profiling Interactive Analysis (GEPIA) online database (http:// gepia.cancer-pku.cn/) was used to analyze the differential expression and survival of candidate genes, and find genes highly expressed in lung cancer samples and significantly related to poor prognosis as the final target genes of key miRNAs.

\section{Vector Construction, Cell Culture, and Luciferase Reporter Gene Assay}

MiR-139-3p (UCUACAGUGCACGUGUCUCCAGU) mimics, miR-139-3p inhibitors (ACUGGAGACACG UGCACUGUAGA), mimics negative control (NC) (UUCUUCGAACGUGUCACGUTT), and inhibitor NC (CAGUACUUUUGUGUAGUACAA) oligonucleotide primers were chemically synthesized (Gene Pharma, Shanghai, China). The 461-bp nucleotide sequence of the downstream noncoding region of $N O B 1$ and the RNA sequence of ABALON were chemically synthesized and constructed between the XhoI and NotI restriction sites of the psiCheck2 vector to construct the NOB1 wild-type 3' untranslated region (UTR) vector and the ABALON wild-type (WT) vector. A point mutation method was used to replace the bases at the binding position of miR-139-3p in NOB1 and ABALON, and the NOB1 mutant-type 3' UTR vector and ABALON mutant-type (MUT) vector were constructed. The NOB1 wild-type 3' UTR vector, NOB1 mutant-type 3' UTR vector, $A B A L O N$ wild-type vector, ABALON mutant-type vector, miR-139-3p mimics, mimic NC, miR-139-3p inhibitors, and inhibitor $\mathrm{NC}$ were transfected in cells at $20 \mathrm{nM}$ using the Lipofectamine 3000 Transfection Reagent (Invitrogen, CA, USA) with serum-free media for $48 \mathrm{~h}$ following the manufacturer's instructions (the amount of plasmids used was $2 \mu \mathrm{g} /$ well). The luciferase reporter assay was performed using the Dual-Luciferase Reporter Assay System (Promega, WI, USA), and the relative luciferase activity was calculated after transfection for $48 \mathrm{~h}$ by normalizing the firefly luminescence to that of Renilla. Each experiment consisted of three technical replicates, and three independent biological replicates were performed.

\section{Statistical Analysis}

Most of the statistical analyses were performed using the aforementioned bioinformatics tools. When differential expression analysis was conducted, only lncRNAs with $\mid \log 2$ fold change $\mid>1$ and $P<0.05$ were considered statistically significant. In the univariate Cox analyses,
$P<0.05$ was regarded as statistically significant for survival analysis and model building; $* P<0.05$, $* * P<0.01$, and ${ }^{* * *} P<0.001$. All calculations and analyses were conducted using the $\mathrm{R}$ software, version 4.0.3.

\section{Results}

\section{Identification of Prognostic- and m6A-Related IncRNAs in Patients with Lung Cancer}

By sorting out and re-annotating TCGA lung cancer expression data, 14,086 lncRNAs were identified. The Pearson correlation analysis showed that 1655 lncRNAs significantly correlated with the expression of m6A genes (Figure 1A). The univariate Cox analysis revealed 22 IncRNAs that were significantly related to the survival of patients with lung cancer (Figure 1B). The KM survival analysis showed that MIR99AHG, SALRNA1, AC007613.1, AC099850.4, AC009690.2, ABALON, AL096701.3, AC087501.4, AL031666.1, AC135050.6, AC104785.1, AC034102.8, SNHG12, and UGDH.AS1 were significantly related to survival (Figure 1C-P).

\section{Prognostic Model Construction and Assessment}

Using the LASSO Cox analysis of 24 m6A-related prognostic lncRNAs, a lung cancer prognostic risk model was constructed containing 14 m6A-related IncRNAs. (Figure 2A-C). Significant survival differences were found between the high- and low-risk groups in the train and test groups, and the Kaplan-Meier survival curves depicted that patients with lung cancer with higher risk scores had worse clinical outcomes (Figure 2D and G). The AUC value of the 1-, 3-, and 5-year ROC curves of the train and test groups was $0.678,0.703,0.672,0.665$, 0.653 , and 0.638 , respectively, demonstrating that the lung cancer prognostic risk model had good predictive accuracy (Figure 2E and $\mathrm{H}$ ). The risk score and survival status distributions are plotted in Figure 2F and I.

\section{Cluster and GSEA Analysis Results of IncRNAs}

When all the samples were divided into two clusters using cluster analysis, the correlation of the internal samples of the cluster was found to be higher and the correlation between the clusters was lower. All samples were divided 
A

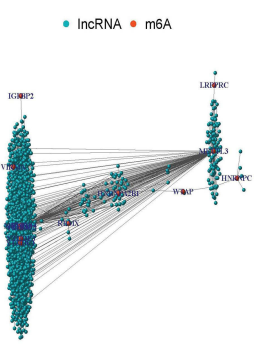

E
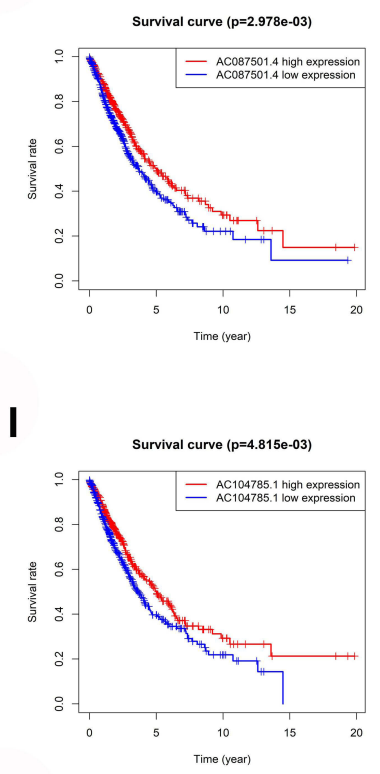

M

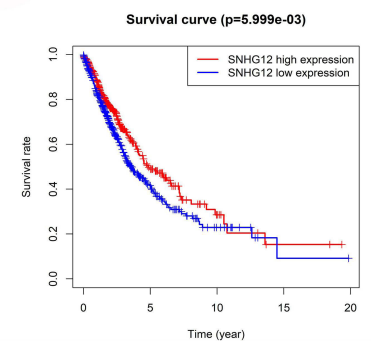

B

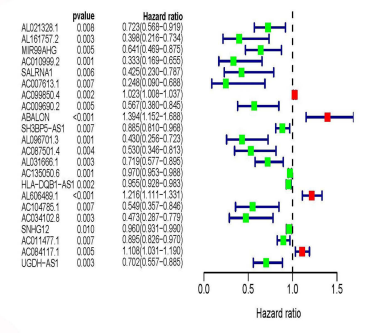

$\mathbf{F}$

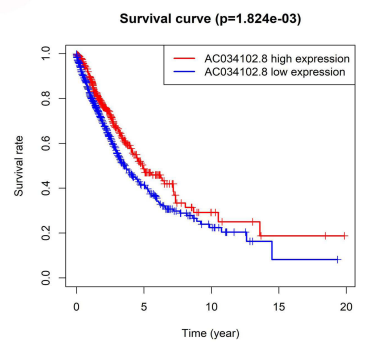

J

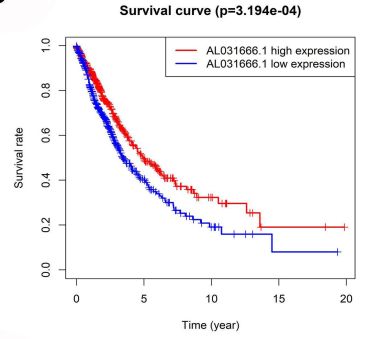

$\mathbf{N}$

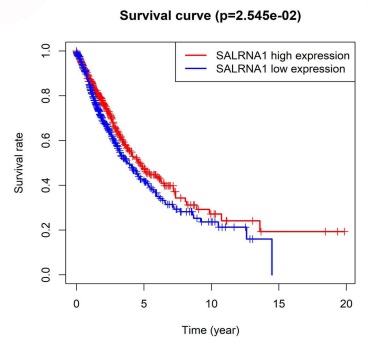

C

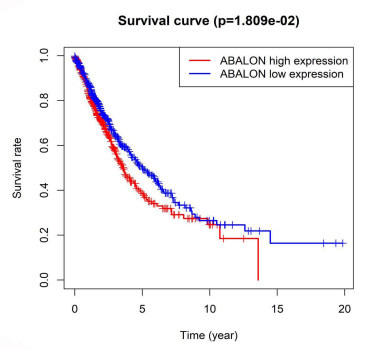

G

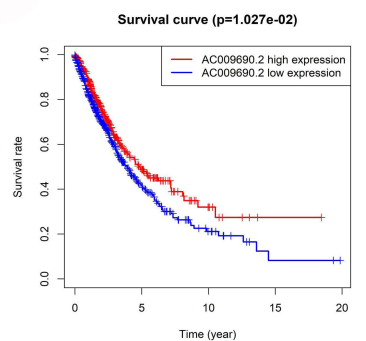

K

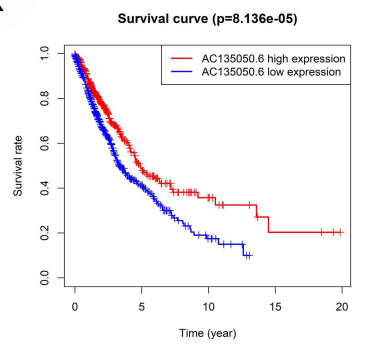

0

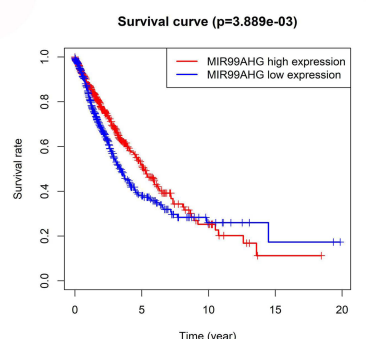

D

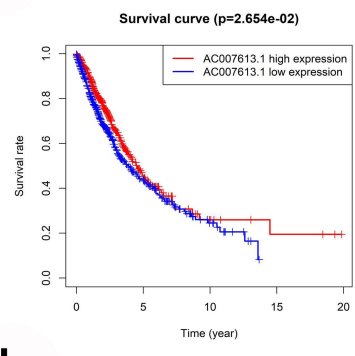

H

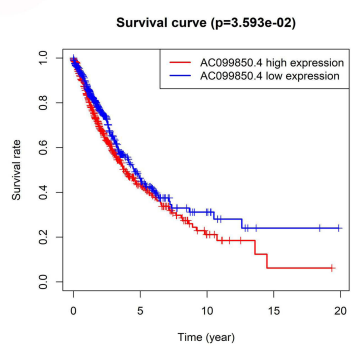

$\mathbf{L}$

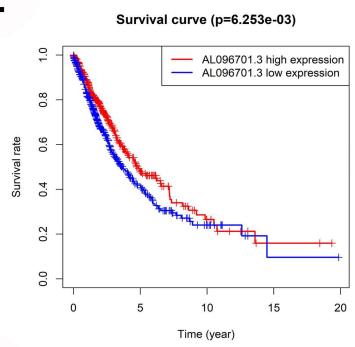

$\mathbf{P}$

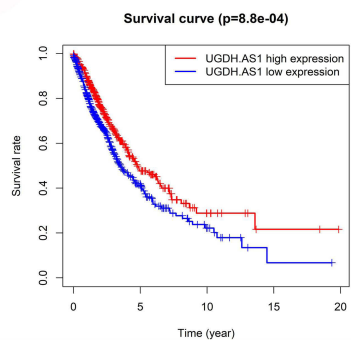

Figure I Mining of m6A-related lung cancer prognostic IncRNAs. (A) Interaction network of m6A genes and IncRNA. (B) m6A-related lung cancer prognostic IncRNAs. (C-P) Survival of ABALON, AC007613.I, AC08750I.4, AC034I02.8, AC009690.2, AC099850.4, ACI04785.I, AL031666.I, ACI35050.6, AL09670I.3, SALRNAI, MIR99AHG and UGDH.ASI.

into two groups, cluster 1 and cluster 2. The clustering graphs of 2 cluster, 3 cluster, 4 cluster, 5 cluster, 6 cluster, 7 cluster, 8 cluster, and the clustering index graphs of 2-8 cluster are plotted in Figure 3A-H. Significant survival differences existed between cluster 1 and cluster 2, and the Kaplan-Meier survival curves depicted that patients with lung cancer in cluster 1 had worse clinical outcomes (Figure 3I). The GSEA revealed that cluster 1 was enriched in signal pathways such as cell cycle, DNA replication, RNA degradation, and so forth. The enrichment of the top 9 sorted is plotted in Figure 4A-I.

\section{Immune Infiltration and Tumor Microenvironment Results}

The analysis of the differences in the content of 22 kinds of immune cells showed 16 kinds of immune 
A

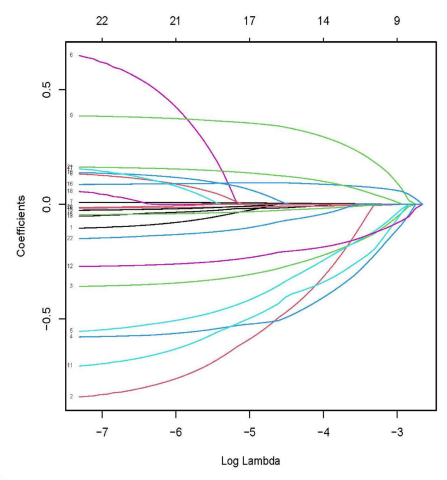

D
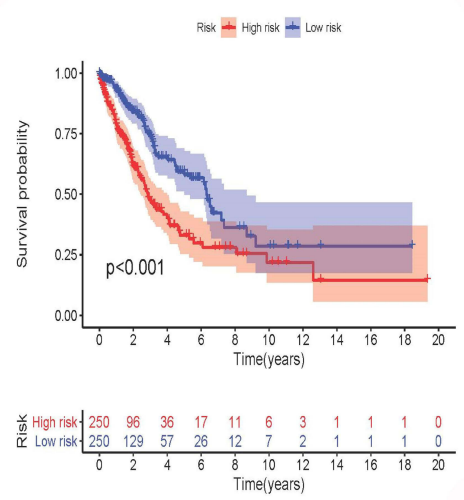

G
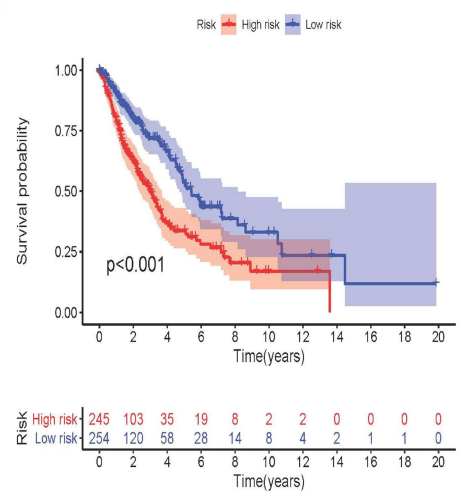

B

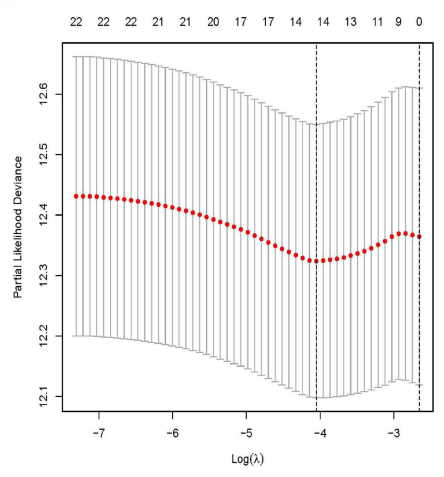

E

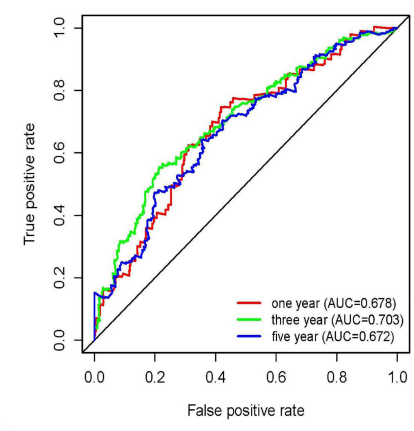

H

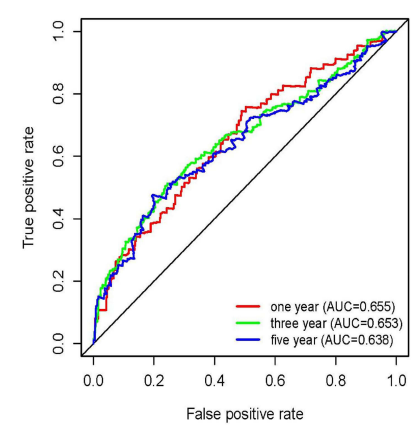

C

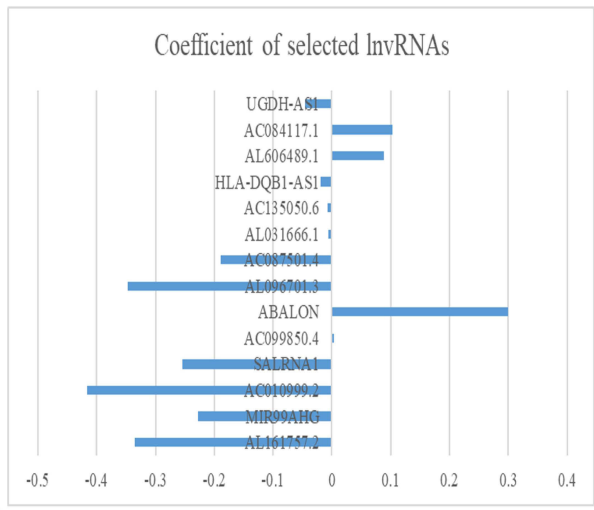

$\mathbf{F}$
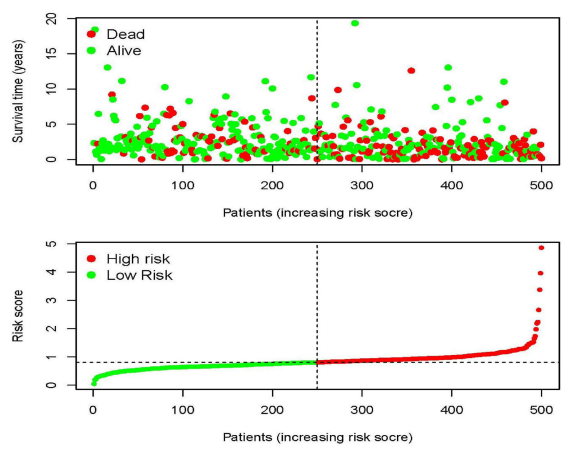

I
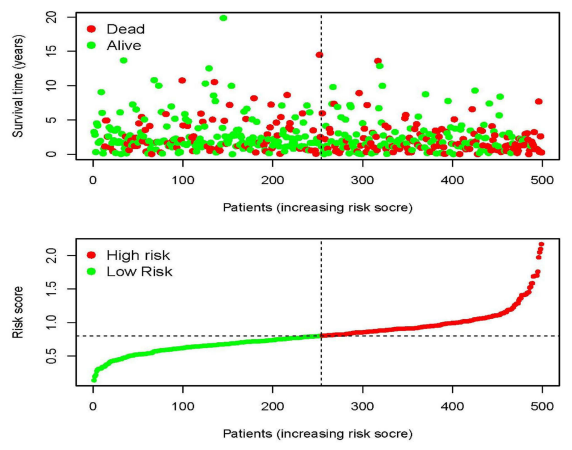

Figure 2 Construction and verification of prognostic risk model. (A and B) Least absolute shrinkage and selection operator (LASSO) regression determines the minimum criteria. (C) Coefficients of selected IncRNAs. (D) Kaplan-Meier curves showed that the high-risk group had worse overall survival than the low-risk group in the train group. (E) The AUC values of the I-, 3-, and 5-year ROC curves of the train group are all greater than 0.6 , indicating that the model has good prediction accuracy. (F) Distributions of risk scores and survival status of lung cancer patients in the train group. (G) Kaplan-Meier curves showed that the high-risk group had worse overall survival than the low-risk group in the test group. $(\mathbf{H})$ The AUC values of the I-, 3-, and 5-year ROC curves of the test group are all greater than 0.6, indicating that the model has good prediction accuracy. (I) Distributions of risk scores and survival status of lung cancer patients in the test group.

cells in cluster 1 and cluster 2 with significant differences (Figure 5A). The immuneScore, stromalScore, and estimateScore of cluster 2 were all higher than those of cluster 1 , indicating that cluster 2 had a better prognosis and higher survival rate than cluster 1 (Figure 5B-D). Correlation analysis showed that 14 kinds of immune cells were significantly related to the risk score of patients with lung cancer (Figure 6A-N). 
A

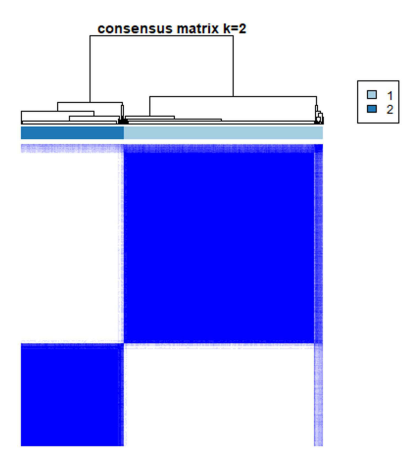

D

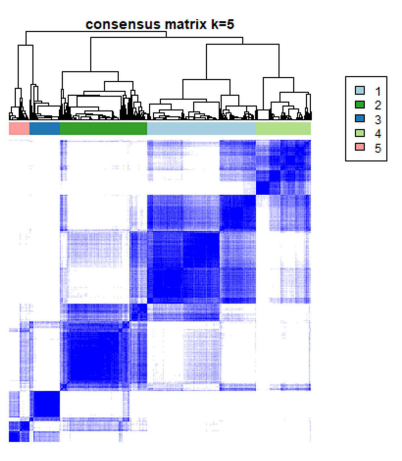

G

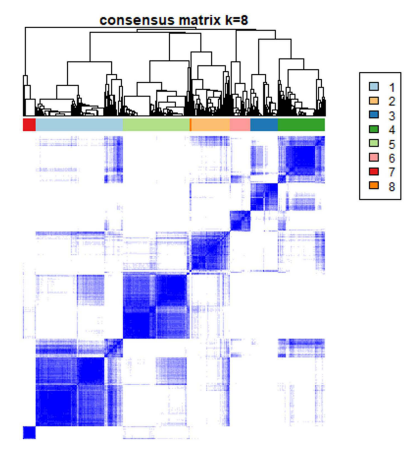

B

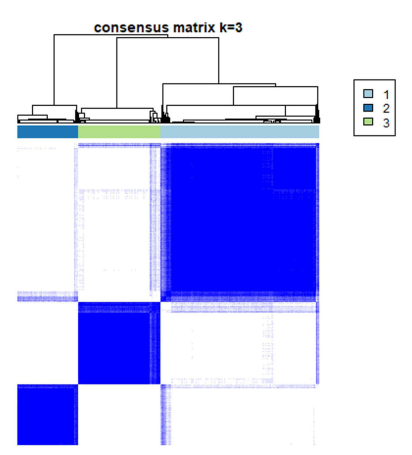

E

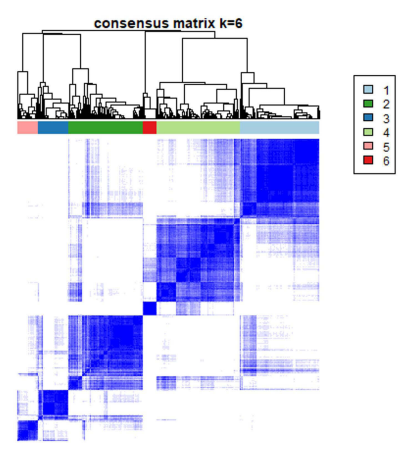

H

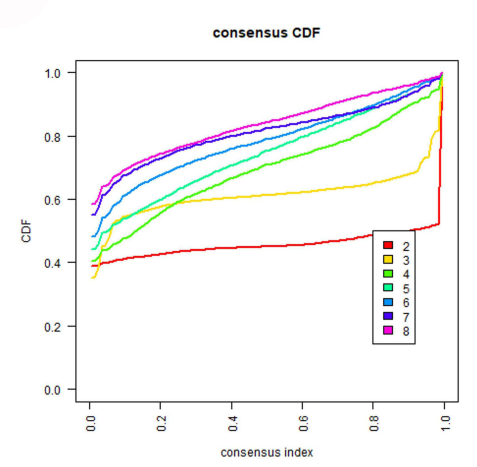

C

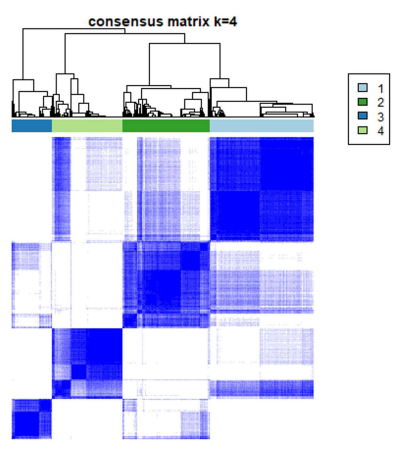

F

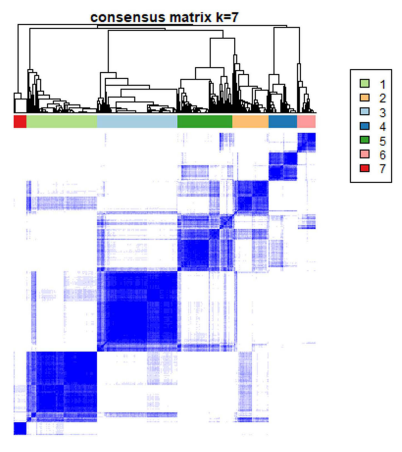

I

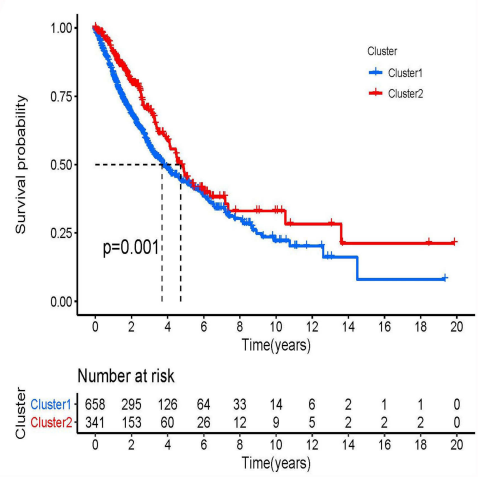

Figure 3 Cluster analysis. (A-G) The clustering graphs of 2-8 cluster. (H) Clustering index graphs. (I) Kaplan-Meier survival curves show cluster I had worse overall survival.

\section{Model IncRNA Differential Expression Analysis}

The differential analysis of lncRNAs in lung cancer and normal samples showed that AC099850.4, SNHG12, ABALON, AC084117.1, AC009690.2, and AL096701.3 were significantly highly expressed in patients with lung cancer (Figure 7A). The differential analysis of lncRNAs in cluster 1 and cluster 2 showed that AC099850.4 and ABALON were significantly highly expressed in cluster 1 (Figure 7B). The differential analysis of lncRNAs in high- and low-risk groups showed that AC099850.4, ABALON, AL606489.1, and AC084117.1 were highly expressed in the high-risk group (Figure 7C). The differential analysis results of the three different groups of lncRNAs showed that AC099850.4 and ABALON may be the most critical.

\section{ABALON Subcellular Localization and Target Prediction}

The prediction results of the lncLocator website showed that ABALON was mainly located in Cytosol, 
A

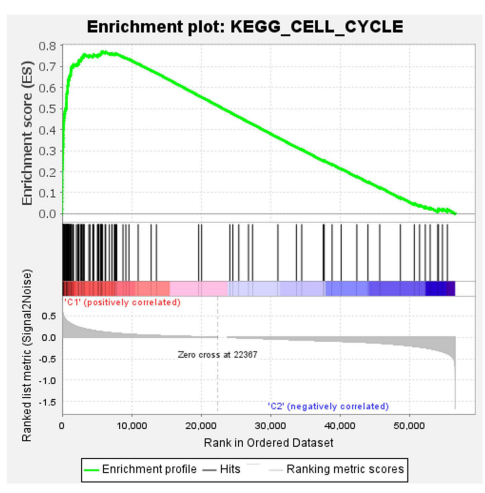

D

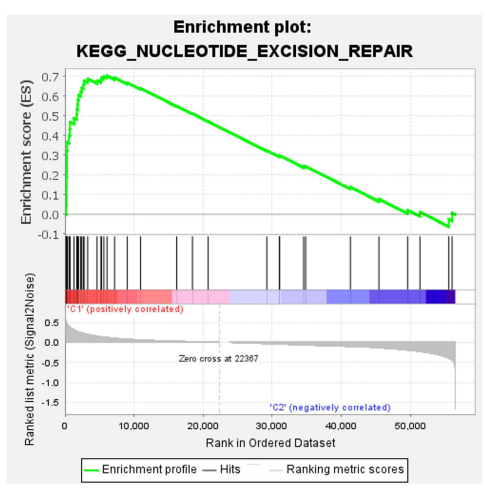

G

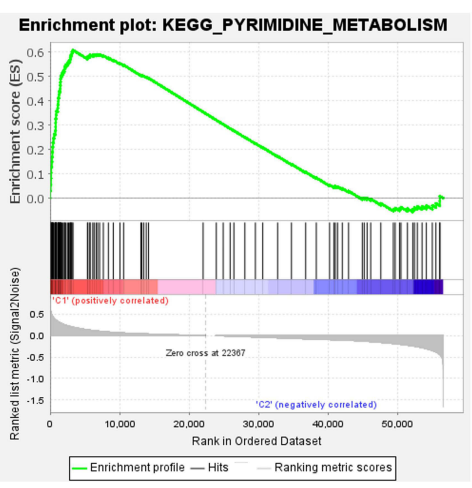

B

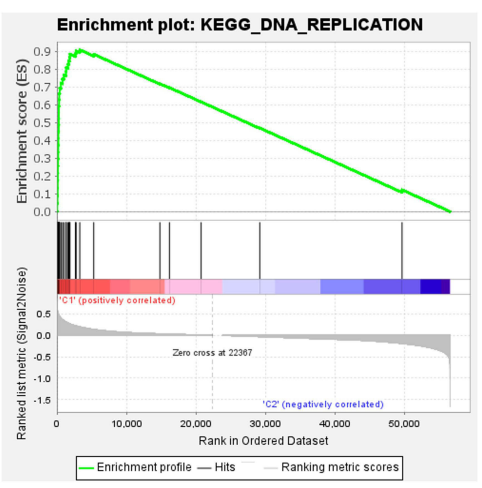

E

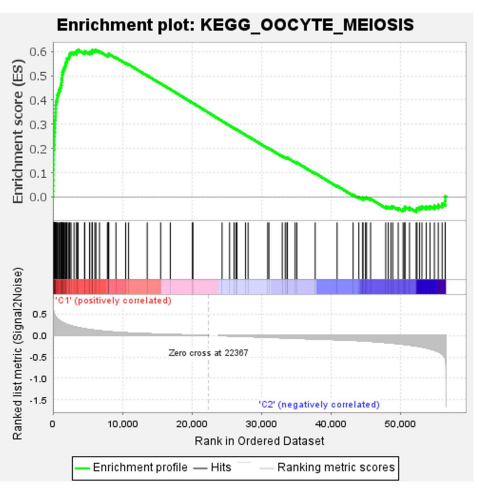

H

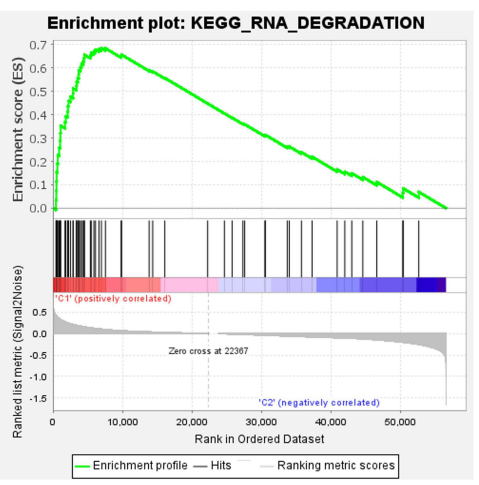

C

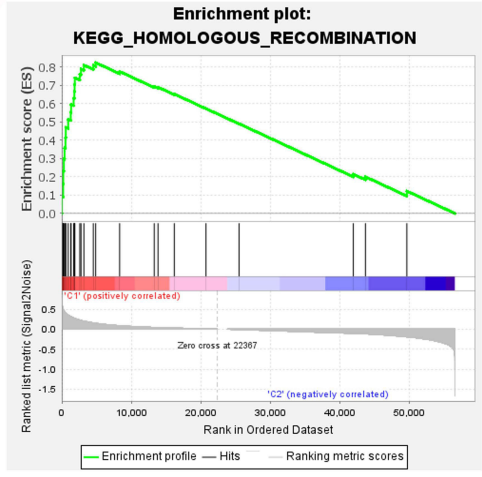

F

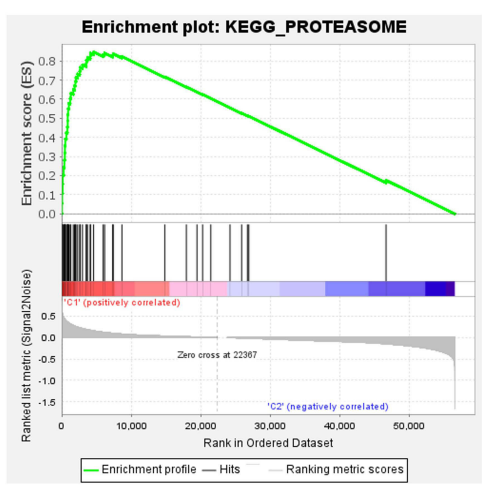

I

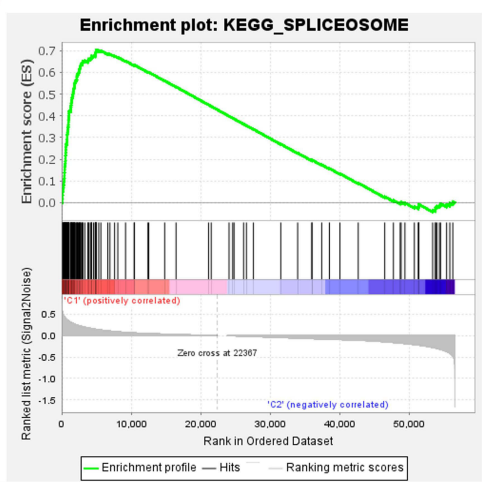

Figure 4 Gene set enrichment analysis. (A) Cell cycle. (B) DNA replication. (C) Homologous recombination. (D) Nucleotide excision repair. (E) Oocyte meiosis. (F) Proteasome. (G) Pyrimidine metabolism. (H) RNA degradation. (I) Spliceosome.

accounting for about $88 \%$ (Figure $8 \mathrm{~A}$ ). The miRDB results showed that 40 miRNAs could bind to ABALON (Figure 8B). The results of differential analysis of miRNAs showed that miR-486-5p, miR-47323p, miR-144-5p, miR-451, miR-139-3p, miR-144-3p, miR-30c-2-3p, miR-490-3p, miR-30a-3p, miR-133b, miR-30a-5p, miR-1-3p, miR-194-3p, miR-133a-3p, miR-143-3p, miR-206, miR-195-5p, miR-139-5p, miR338-5p, miR-218-1-3p, miR-218-5p, miR-4529-3p, and
miR-190a-5p were highly expressed in the lung of normal patients (Figure 8D). Only one intersection was present between these highly expressed miRNAs and the ABALON target miRNAs, that is, miR-139-3p; therefore, miR-139-3p was selected as the key miRNA. The three databases miRDB, miRTarBase, and TargetScan all showed that miR-139-3p could specifically bind to VWA1, MIEF1, NOB1, CCDC71L, FN3K, UBE2G1, and ELAVL1 (Figure 8C). 
A
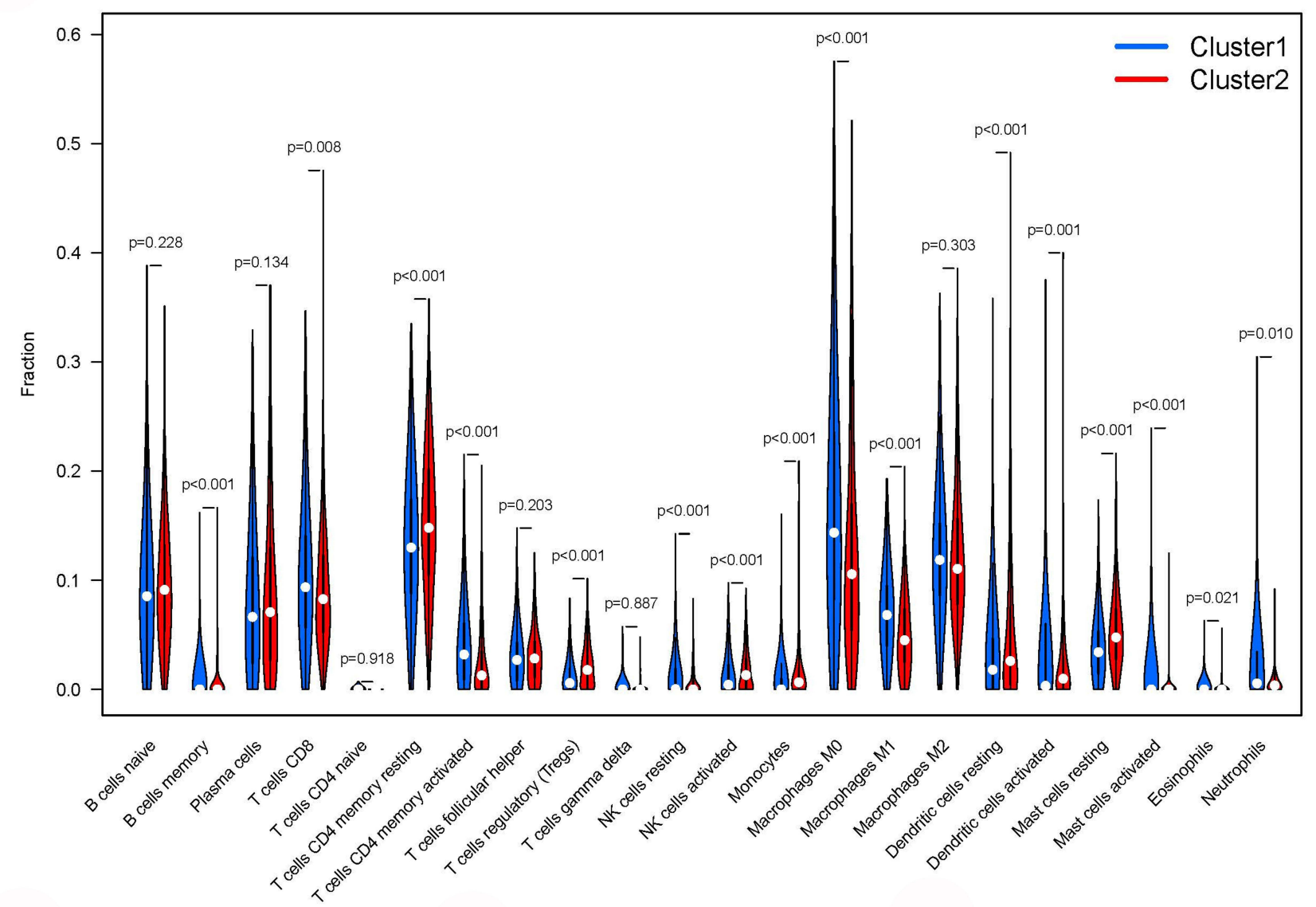

B

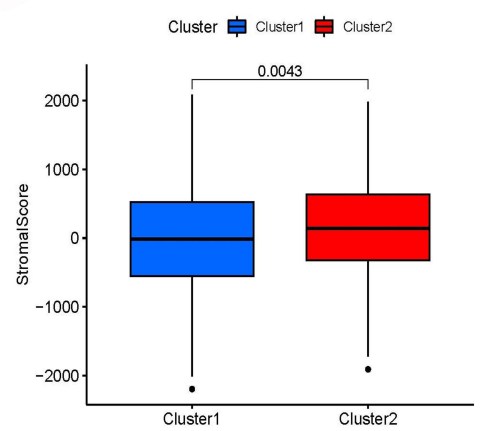

C

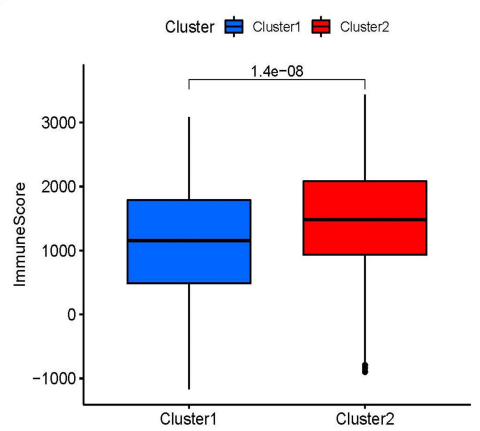

D

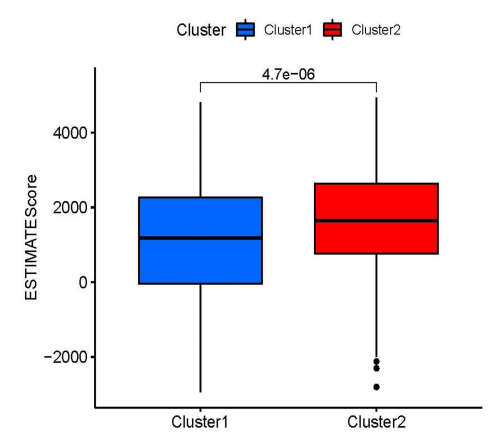

Figure $\mathbf{5}$ Immune infiltration and tumor microenvironment. (A) The expression of 22 immune cells between cluster I and cluster 2. (B) The immuneScore (C) stromalScore (D) eSTIMATEScore indicating that cluster I had a worse overall survival than cluster 2.

\section{Differential Analysis and Survival Analysis of miR-139-3p Target Genes}

The differential analysis and survival analysis of miR-139$3 \mathrm{p}$ by GEPIA showed that ELAVL1, MIF1, NOB1, and $V W A 1$ were highly expressed in patients with lung cancer (not significant). Also, NOB1 and UBE2G1 were significantly related to the survival of patients with lung cancer, and the high expression of $N O B 1$ and $U B E 2 G 1$ led to a low survival rate and poor prognosis (Figure 9A-N).
NOB1 was selected as the target gene of miR-139-3p, and the combination of ABALON, miR-139-3p, and NOB1 is plotted in Figure 9O.

ABALON Acted as a ceRNA by Sponging miR-I39-3p and Indirectly Regulated the

\section{Expression of NOBI}

The luciferase reporter gene assay results showed that cells were cotransfected with ABALON-WT and miR-139-3p 
A

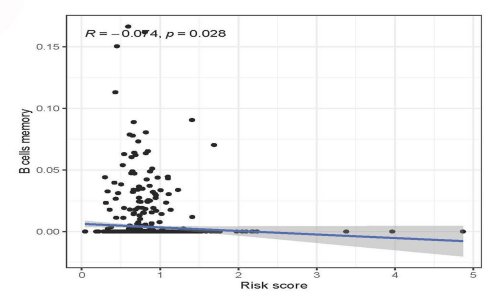

D

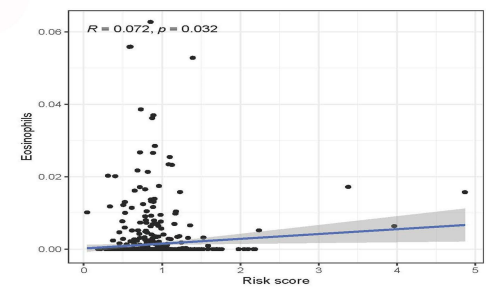

G

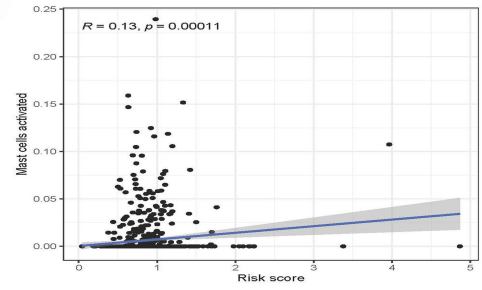

J

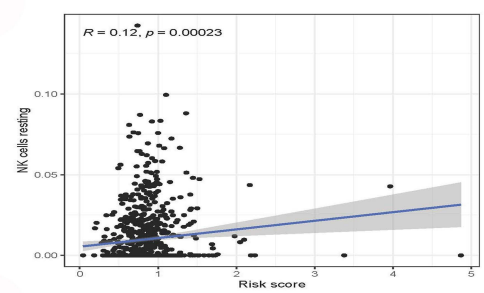

M

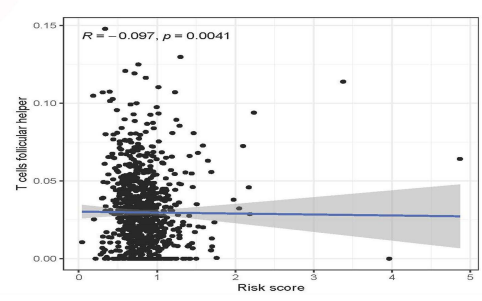

B

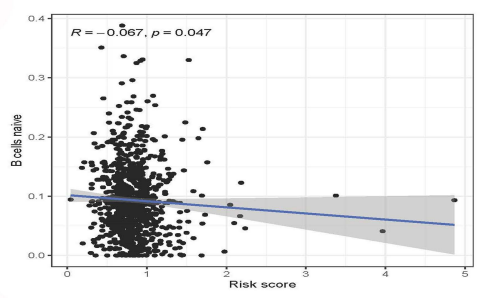

E

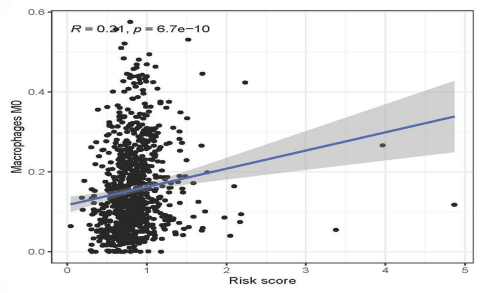

H

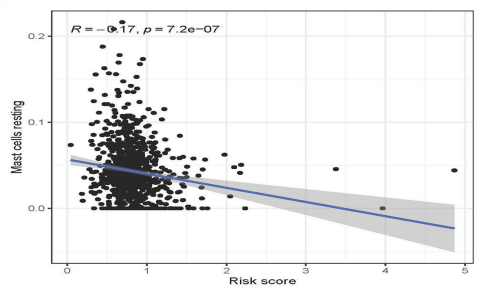

K

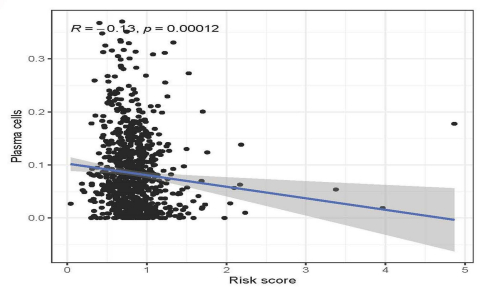

$\mathbf{N}$

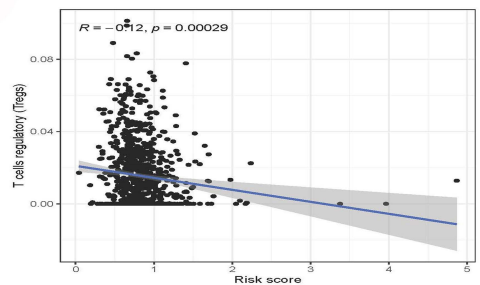

C

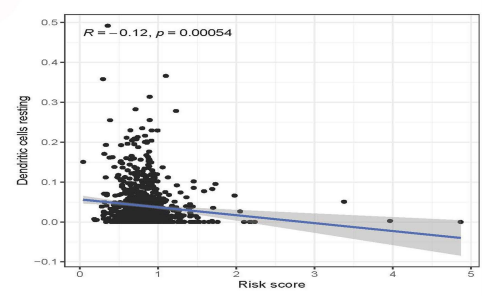

F

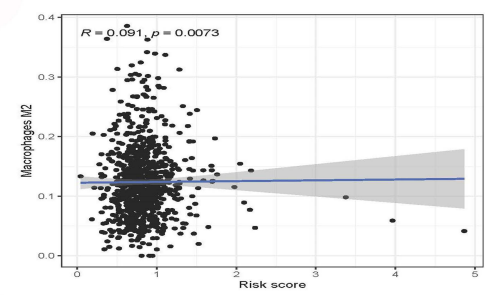

I

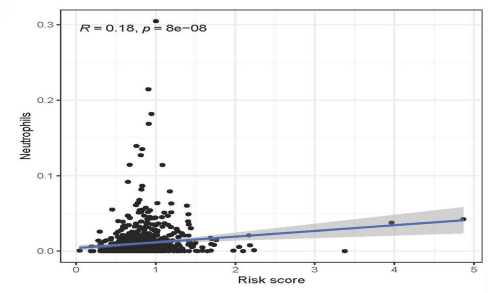

$\mathbf{L}$

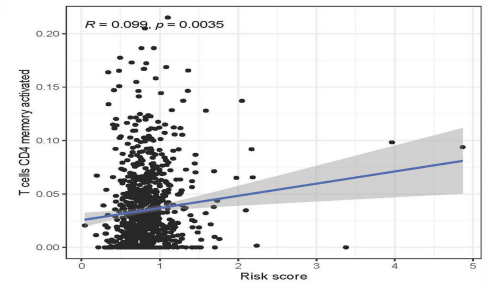

Figure 6 Correlation analysis showed that 14 kinds of immune cells are significantly related to the risk score of lung cancer patients. (A) B cells memory. (B) B cells naive. (C) Dendritic cells resting. (D) Eosinophils. (E) Macrophages M0. (F) Macrophages M2. (G) Mast cells activated. (H) Mast cells resting. (I) Neutrophils. (J) NK cells resting. (K) Plasma cells. (L) T cells CD4 memory activated. (M) T cells follicular helper. (N) T cells regulatory (Tregs).

mimics, which resulted in a significantly reduced activity of the luciferase reporter gene, yet the luciferase activity was not significantly attenuated in the target region of the mutated ABALONR-MUT construct (Figure 10A). When cotransfected with ABALON-WT and miR-139-3p inhibitors, the activity of the luciferase reporter gene significantly upregulated, but the ABALONR-MUT construct had no such change (Figure 10B). The luciferase reporter gene assay results also showed that miR-139-3p mimics could bind to and inhibit the expression of $N O B 1$ (Figure 10C), while miR139-3p inhibitors could eliminate this effect (Figure 10D).

\section{Discussion}

Lung cancer is the most leading cause of cancer deaths in men worldwide, especially in China. Despite recent advances in diagnostics and treatment, the prognosis of 
A

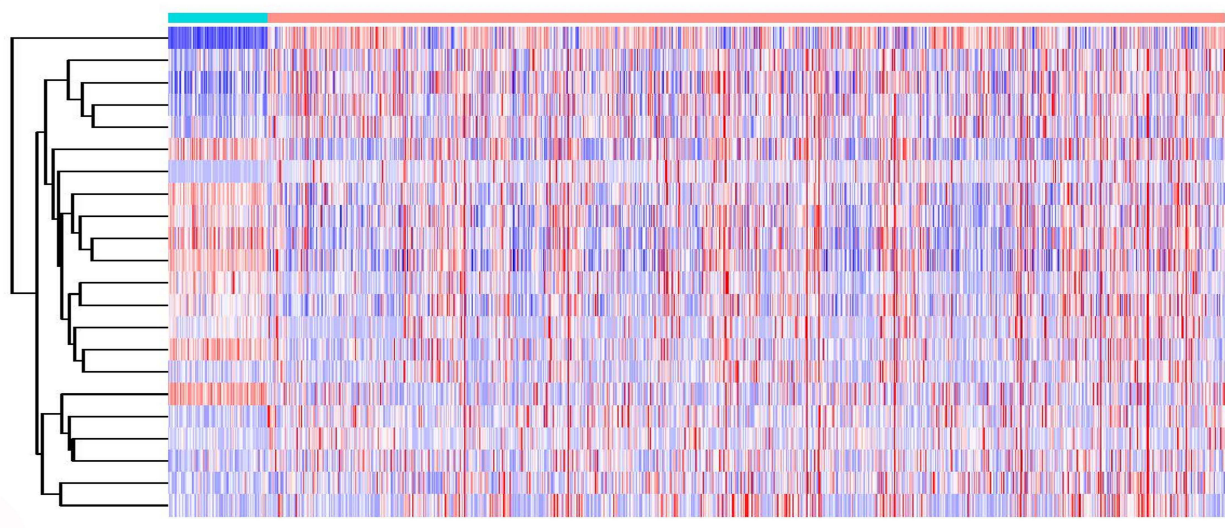

TYP.

AC084117.1*

SNHG12"N*

AC00960. $2^{*}$

HLA-DOB1-AS1.

SALRNA $1 . .$.

AC087501.4**

AC011477. $1^{\text {*A }}$

SH3BP5-AS1**

AC135050.6*"

AC007613.1*

UGDH-AST.

ALO21328.1\%

AC0 $34102.8^{* *}$

MIRG9AHO

ACoros99.2

AC104785. 1*

ABALON ${ }^{* * *}$.

AL606489. $1^{\text {N. }}$
B

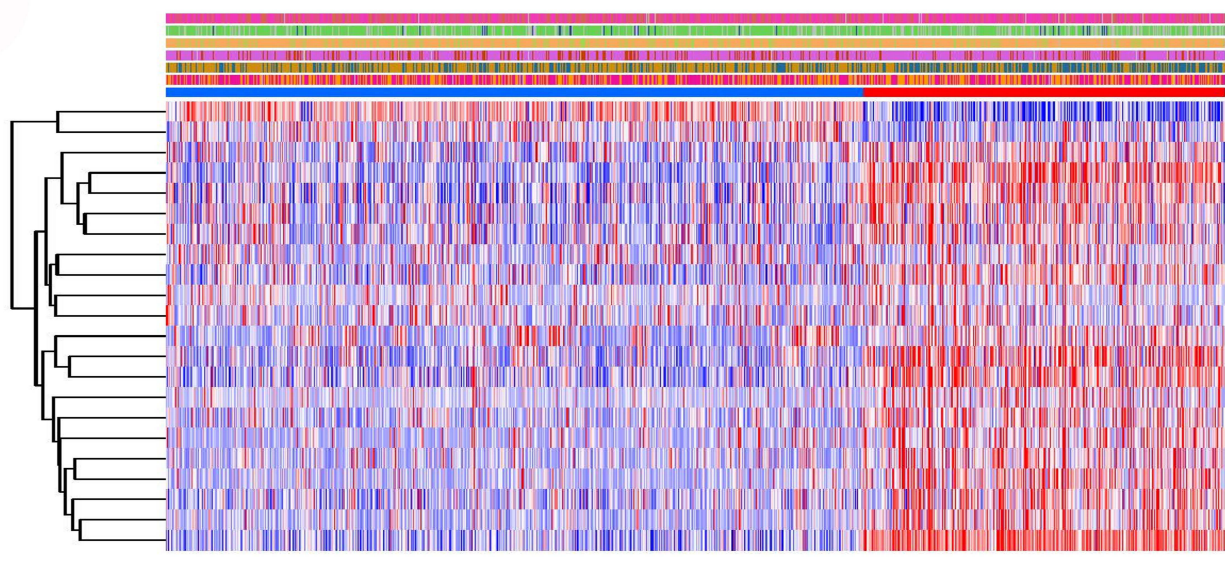

$N^{\infty \times x}$
$\mathbf{M}^{n}$
Stage:

Trage
Standern
Gene
Age

AC099850.4

ACO84117.1

SNHG12

AC009690.

AL096701.3

AC087501.4

AL161757.2

A.010000.

HLA-DOB1-AS

ACO11477.

AC104785.

ALO21328.1

Co07613.

C

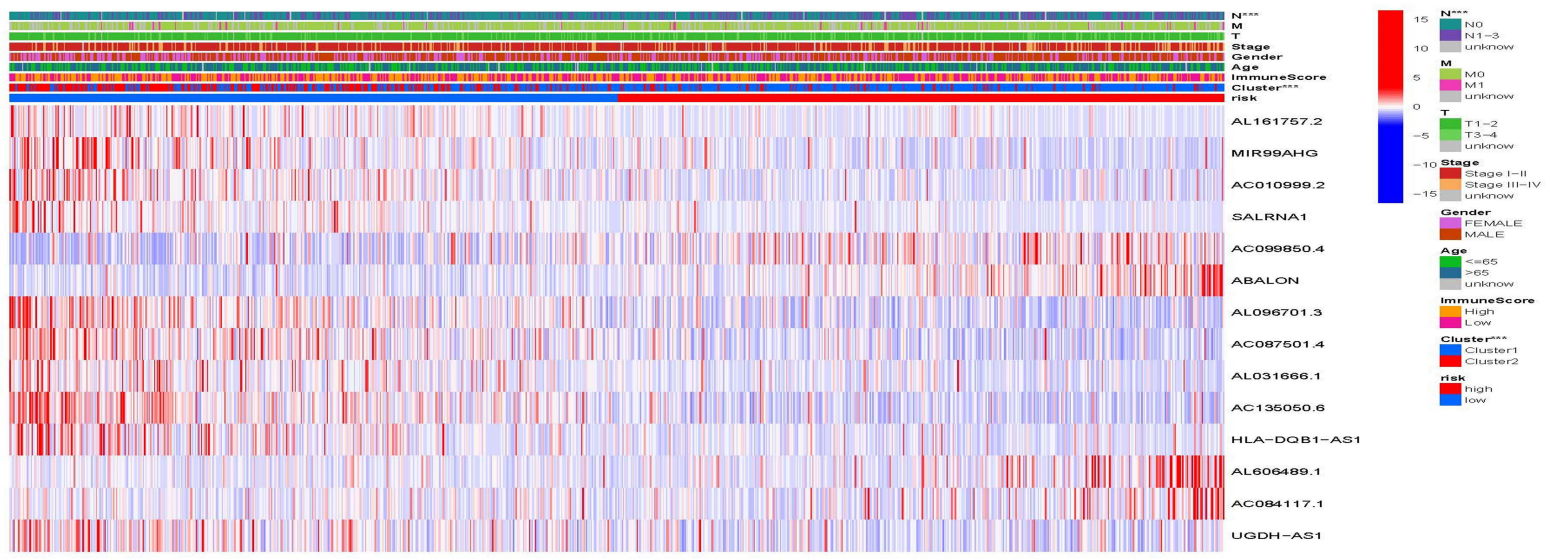

Figure 7 Model IncRNA differential expression. (A) AC099850.4, SNHG I2, ABALON, AC084I I7.I, AC009690.2 and AL09670I.3 were significantly high expression in lung cancer patients. (B) AC099850.4 and ABALON were significantly highly expressed in cluster I. (C) AC099850.4, ABALON, AL606489.I and AC084II7.I were highly expressed in high-risk group.

patients with lung cancer remains poor. ${ }^{26,27}$ Most patients were diagnosed with distant metastasis, which was often accompanied by a poor prognosis, and the pathogenesis of lung cancer at the molecular level was not clear. ${ }^{28,29}$ Therefore, the identification of more effective biomarkers for diagnosis and treatment was urgently needed.

In this study, by integrating the expression data and clinical information of 1037 cancer samples and 108 normal samples, a co-expression analysis of $23 \mathrm{~m} 6 \mathrm{~A}$ genes and 14,086 IncRNAs was performed, an m6A gene-lncRNA co-expression network was constructed, and 1655 m6A-related IncRNAs were determined in patients with lung cancer. Using univariate Cox analysis and LASSO Cox analysis, this study constructed a lung cancer prognostic risk model based on m6A-related lncRNA. The AUC values of the 1-, 3-, and 5-year ROC 
A

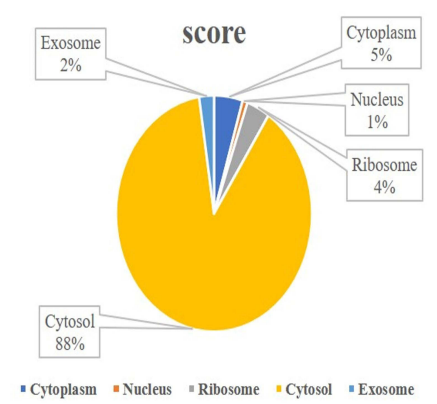

B

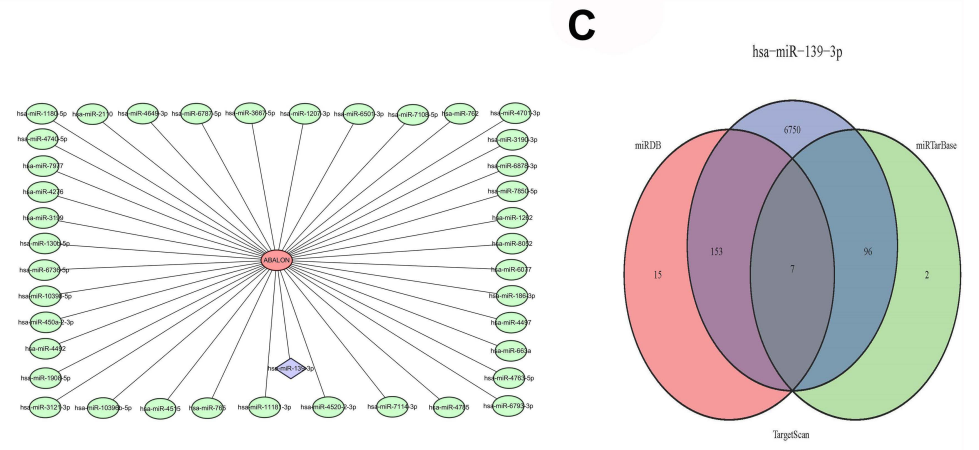

D

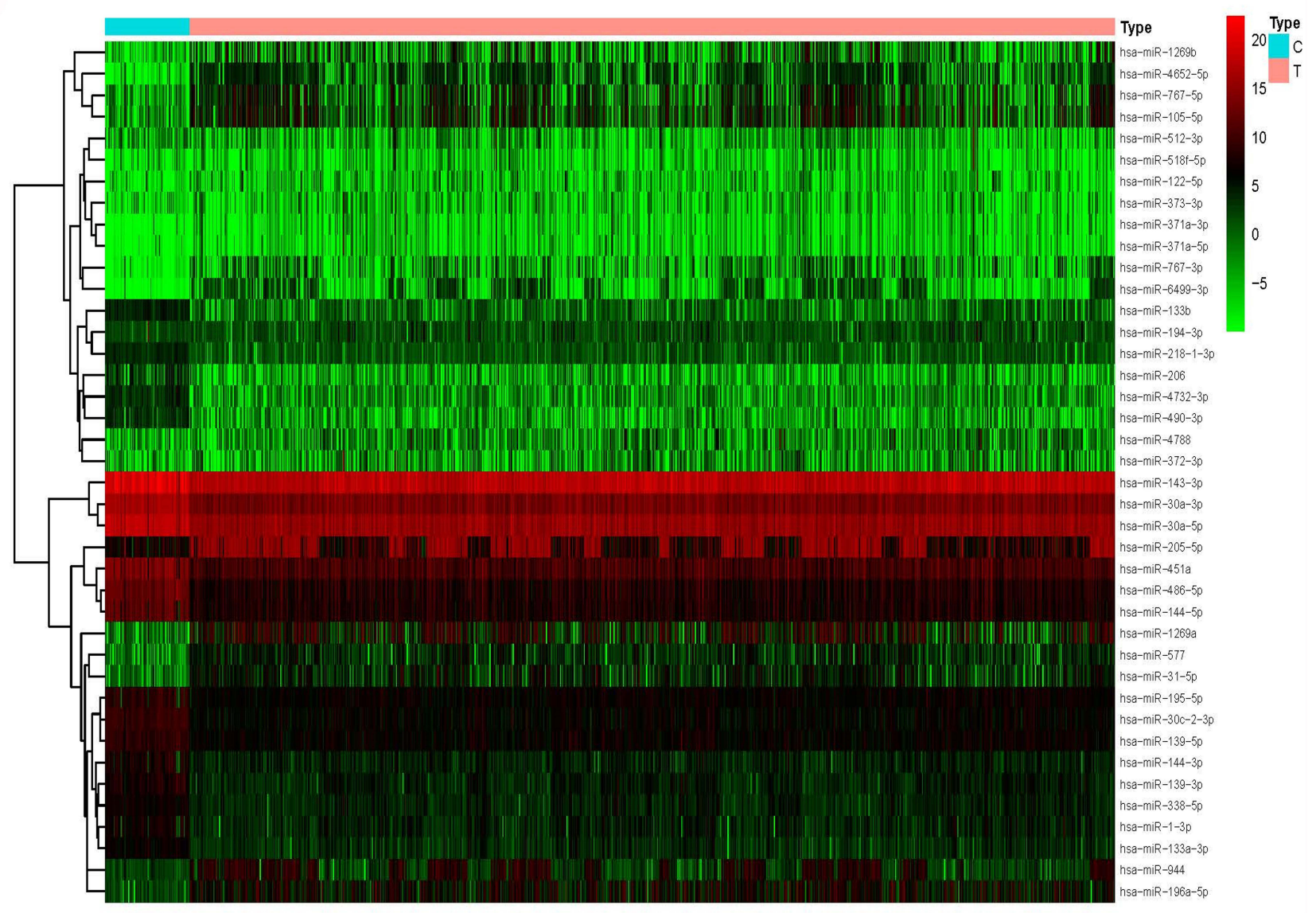

Figure 8 Subcellular localization and target prediction of IncRNA ABALON. (A) The subcellular localization results show that ABALON is mainly enriched in Cytosol. (B) The miRDB results showed that 40 miRNAs can bind to ABALON. (C) VWAI, MIEFI, NOBI, CCDC7IL, FN3K, UBE2GI and ELAVL are the target genes of miR-I39-3p. (D) miR-486-5p, miR-4732-3p, miR-I44-5p, miR-45I, miR-139-3p, miR-I44-3p, miR-30c-2-3p, miR-490-3p, miR-30a-3p, miR-133b, miR-30a-5p, miR-I-3p, miR-I94-3p, miR-133a-3p, miR-I43-3p, miR-206, miR-195-5p, miR-139-5p, miR-338-5p, miR-2I8-I-3p, miR-218-5p, miR-4529-3p and miR-190a-5p were highly expressed in lung cancer normal patients.

curves of the train and test groups were all greater than 0.06 , demonstrating that the prognostic risk model had good predictive accuracy. Cluster analysis, immune analysis, and GSEA analysis showed that the IncRNAs of the lung cancer prognostic risk model constructed was mainly involved in cell cycle, DNA replication, RNA degradation, and other biological processes, and was significantly related to immunity. This implied that the concerned IncRNAs may participate in the occurrence of lung cancer by regulating the cell cycle and RNA expression.

ABALON is an antisense long noncoding RNA of apoptotic BCL2L1, with a length of 1903 bp, mainly enriched in Cytosol. ${ }^{30}$ Currently, no study has reported on ABALON. The average expression of ABALON in 
A

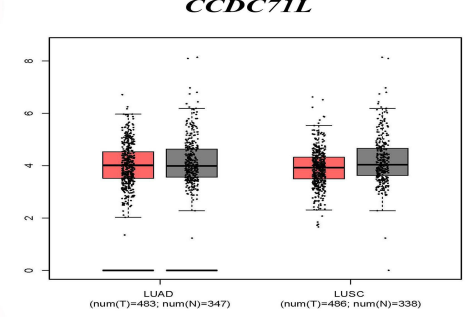

D

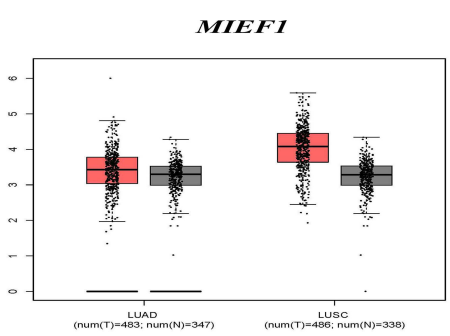

G

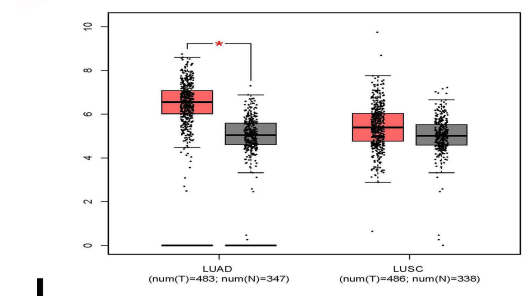

J

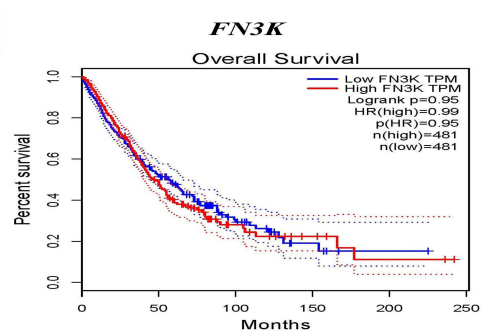

M

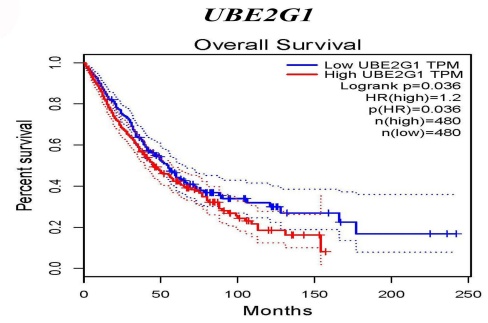

B

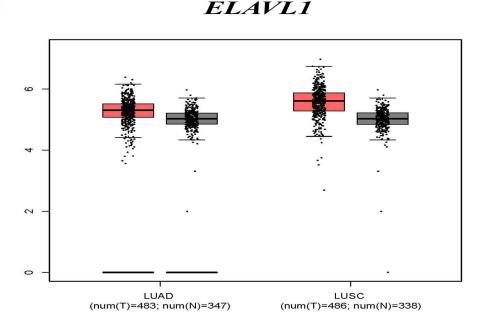

E

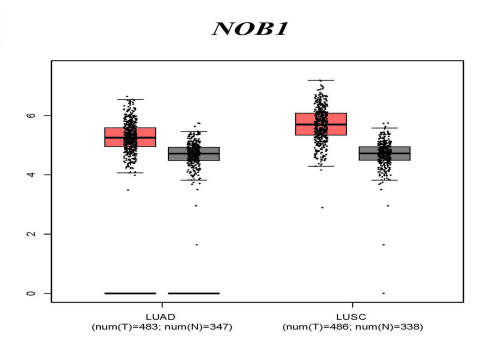

H

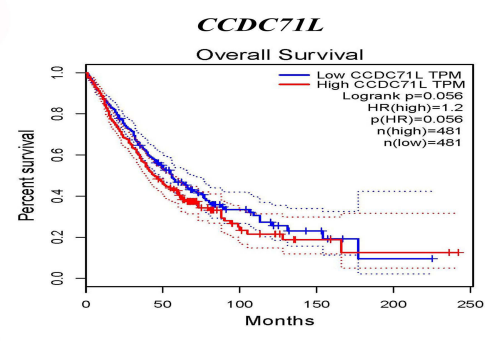

K

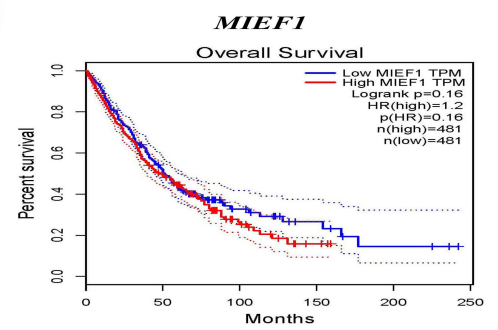

$\mathbf{N}$

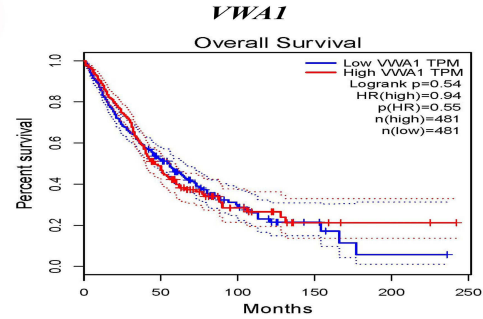

C

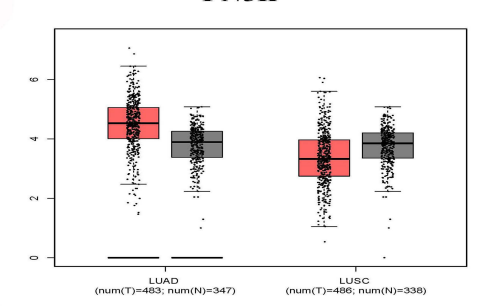

F

UBE2GI

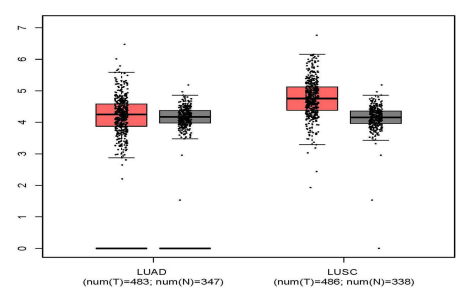

I

ELAVEI

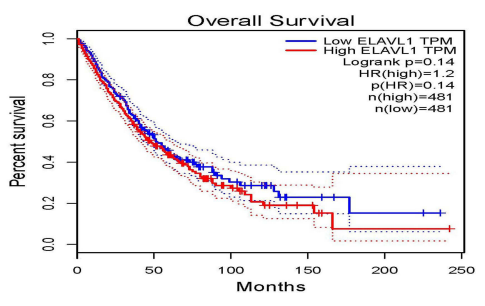

L

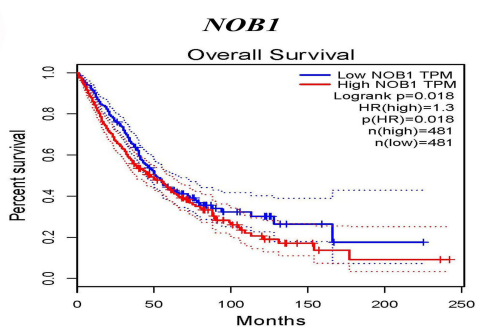

0

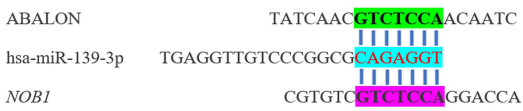

Figure 9 Differential expression and survival analysis of target genes. (A-G) The expression of CCDC7IL, ELAVL, FN3K, MIEFI, NOBI, UBE2GI, VWAI between lung cancer samples and normal samples. (H-N) Kaplan-Meier survival curves of CCDC7IL, ELAVL, FN3K, MIEFI, NOBI, UBE2GI, VWAI between lung cancer samples and normal samples. (O) ABALON-miR-I39-3p-NOBI base complementary binding situation.

the patients with lung cancer was higher than that of normal samples, although the difference was not significant. Patients with lung cancer with high expression of ABALON had a lower survival rate and poor prognosis. This implied that ABALON was important in the occurrence of lung cancer. Studies have shown that miR-139-3p could inhibit the proliferation, migration, and invasion of a variety of cancer cells, ${ }^{31-36}$ including lung cancer. ${ }^{37}$ The high expression of miR-139-3p also significantly positively correlated with a better cancer prognosis. ${ }^{38}$ In vivo and in vitro studies have shown that $N O B 1$ could promote the proliferation and tumor growth of lung cancer cells. ${ }^{39,40}$ The ABALON-miR-139-3p-NOB1 ceRNA regulatory network was excavated and constructed through bioinformatics methods. It was experimentally verified that ABALON acted as a ceRNA by sponging miR-139- 
A

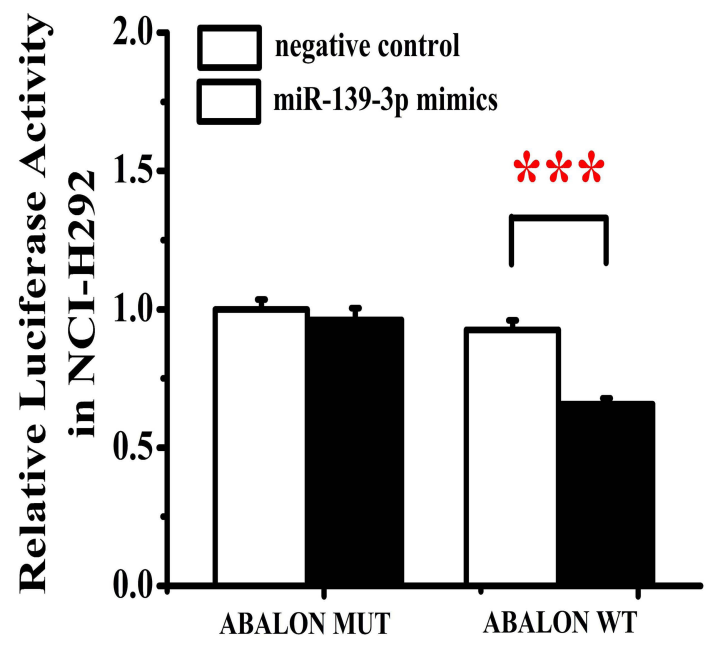

C

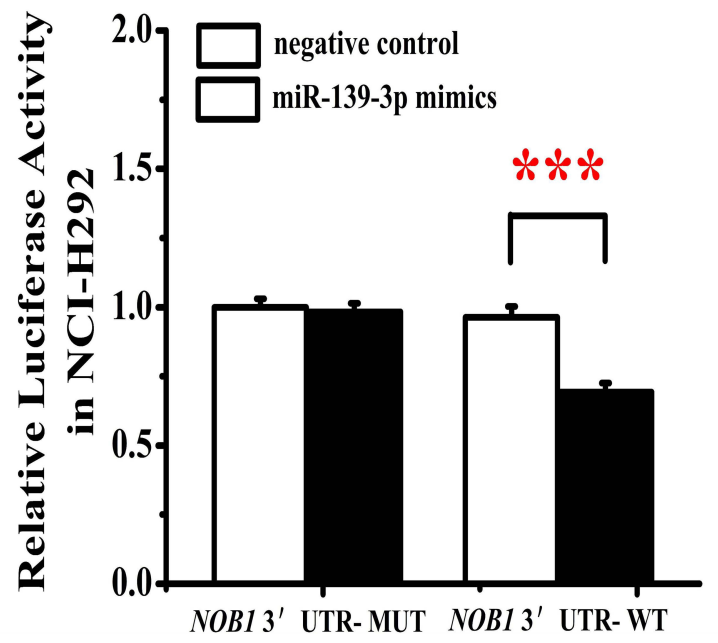

B
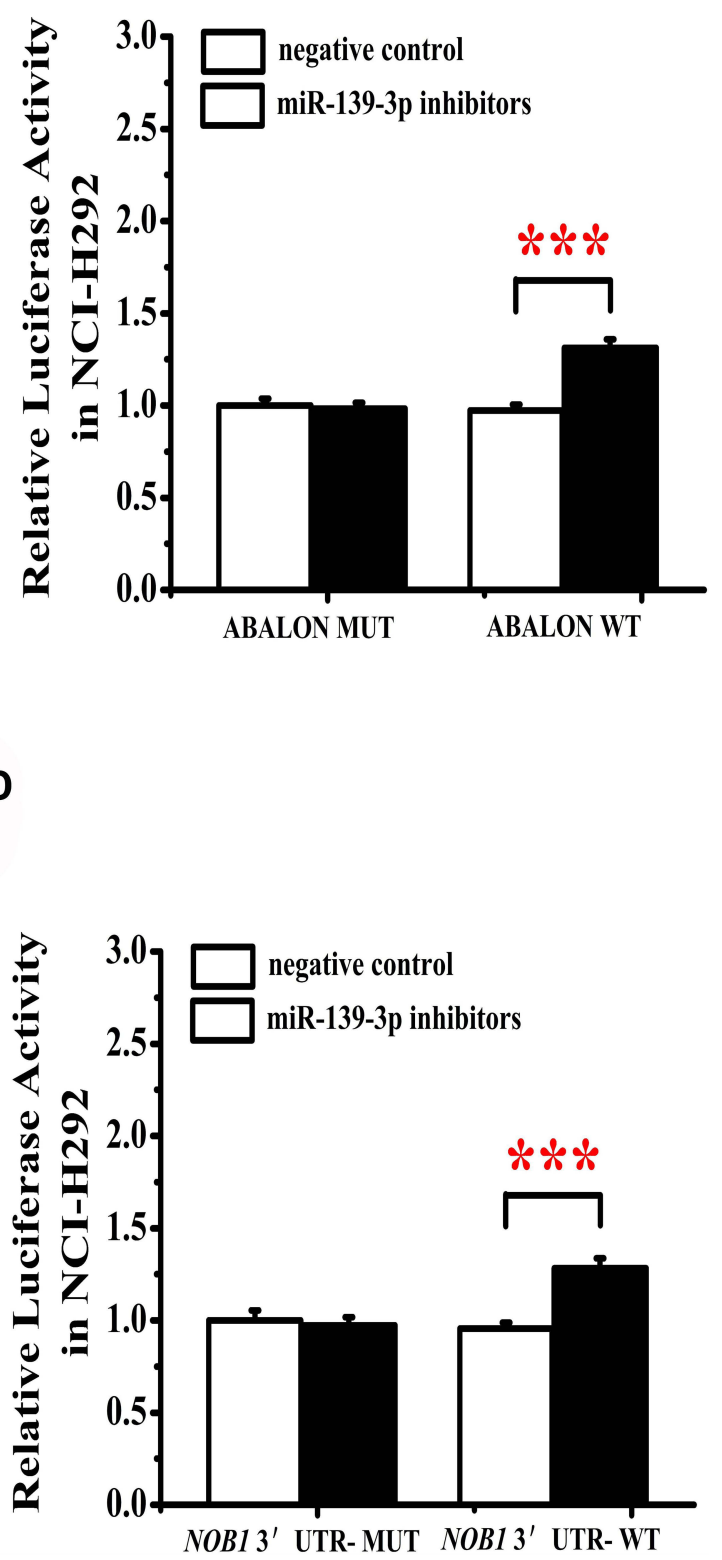

Figure 10 miR-139-3p targets ABALON and NOBI. (A) MiR-I39-3p mimics target binding and reduce ABALON expression. (B) MiR-I39-3p inhibitors can increase the activity of ABALON. (C) MiR-I39-3p mimics target binding and reduce NOBI expression. (D) MiR-I39-3p inhibitors can increase the activity of NOBI. ***<0.00I.

$3 \mathrm{p}$ and indirectly regulated $N O B 1$ expression through the luciferase report experiment. These experimental results further increased the reliability of the lung cancer lncRNA prognostic risk model constructed in this study and provided new ideas for the research on the occurrence and development of lung cancer.

Although a large number of sample data has been found and integrated bioinformatics analysis of the lung cancer IncRNA and miRNA was conducted, still many deficiencies exist. On the one hand, the subcellular location of ABALON was predicted through the lncLocator online website. The ABALON fluorescence in situ hybridization should be performed to evaluate the subcellular localization of IncRNA more accurately. On the other hand, the effect of ABALON could not be verified on lung cancer cell proliferation and tumor formation in vivo and in vitro, which should be an important part of future studies. 


\section{Conclusions}

In summary, this study constructed an m6A-related IncRNA lung cancer prognostic risk model by analyzing the expression data and clinical data of TCGA lung cancer samples. It was experimentally verified that ABALON acted as a ceRNA by sponging miR-139-3p and indirectly regulated NOB1 expression. This study provided a new biological target for the early diagnosis of lung cancer and a new direction for studying the mechanism of lung cancer.

\section{Ethical Statement}

This study protocol was approved by the Ethics Committee of Chongqing Collaborative Innovation Center for Functional Food (2021041001H, Chongqing, China).

\section{Funding}

This research was funded by Chongqing University Innovation Research Group Project (CXQTP20033), the Science and Technology Project of Chongqing Education Commission (KJQN202001604), and Natural Science Foundation of Chongqing Science and Technology Commission (cstc2020jcyj-msxmX0874), China.

\section{Disclosure}

The authors declare that they have no conflicts of interest.

\section{References}

1. Chen R, Xu X, Qian Z, et al. The biological functions and clinical applications of exosomes in lung cancer. Cell Mol Life Sci. 2109;76 (23):4613-4633. doi:10.1007/s00018-019-03233-y

2. Quintanal-Villalonga Á, Molina-Pinelo S. Epigenetics of lung cancer: a translational perspective. Cell Oncol. 2019;42(6):739-756. doi:10.1007/s13402-019-00465-9

3. Han W, Ren X, Yang Y, et al. microRNA-100 functions as a tumor suppressor in non-small cell lung cancer via regulating epithelialmesenchymal transition and Wnt/ $\beta$-catenin by targeting HOXA1. Thorac Cancer. 2020;11(6):1679-1688. doi:10.1111/1759-7714.13459

4. Reck M, Heigener DF, Mok T, et al. Management of non-small-cell lung cancer: recent developments. Lancet. 2013;382(9893):709-719. doi:10.1016/S0140-6736(13)61502-0

5. Forde PM, Brahmer JR, Kelly RJ. New strategies in lung cancer: epigenetic therapy for non-small cell lung cancer. Clin Cancer Res. 2014;20(9):2244-2248. doi:10.1158/1078-0432.CCR-13-2088

6. Paraskevopoulou MD, Hatzigeorgiou AG. Analyzing miRNA-lncRNA interactions. Methods Mol Biol. 2106;1402:271-286. doi:10.1007/9781-4939-3378-5_21

7. Huang Y. The novel regulatory role of lncRNA-miRNA-mRNA axis in cardiovascular diseases. J Cell Mol Med. 2018;22(12):5768-5775. doi: $10.1111 / \mathrm{jcmm} .13866$

8. Ambros V. The functions of animal microRNAs. Nature. 2004;431 (7006):350-355. doi:10.1038/nature02871
9. Tutar Y. miRNA and cancer; computational and experimental approaches. Curr Pharm Biotechnol. 2014;15(5):429. doi:10.2174/ 138920101505140828161335

10. Zhao Y, Feng C, Li Y, et al. LncRNA H19 promotes lung cancer proliferation and metastasis by inhibiting miR-200a function. $\mathrm{Mol}$ Cell Biochem. 2019;460(1-2):1-8. doi:10.1007/s11010-01903564-1

11. $\mathrm{Xu} \mathrm{Y,} \mathrm{Lin} \mathrm{J,} \mathrm{Jin} \mathrm{Y,} \mathrm{et} \mathrm{al.} \mathrm{The} \mathrm{miRNA} \mathrm{hsa-miR-6515-3p} \mathrm{potentially}$ contributes to IncRNA H19-mediated-lung cancer metastasis. J Cell Biochem. 2019;120(10):17413-17421. doi:10.1002/jcb.29006

12. Wang M, Mao C, Ouyang L, et al. Long noncoding RNA LINC00336 inhibits ferroptosis in lung cancer by functioning as a competing endogenous RNA. Cell Death Differ. 2019;26(11):2329-2343. doi:10.1038/s41418-019-0304-y

13. Pan J, Fang S, Tian H, et al. IncRNA JPX/miR-33a-5p/Twist1 axis regulates tumorigenesis and metastasis of lung cancer by activating Wnt/ $\beta$-catenin signaling. Mol Cancer. 2020;19(1):9. doi:10.1186/ s12943-020-1133-9

14. Zhen Q, Gao LN, Wang RF, et al. LncRNA DANCR promotes lung cancer by sequestering miR-216a. Cancer Control. 2018;25 (1):107327481876984. doi:10.1177/1073274818769849

15. Zhao BS, Roundtree IA, He C. Post-transcriptional gene regulation by mRNA modifications. Nat Rev Mol Cell Biol. 2017;18(1):31-42. doi:10.1038/nrm.2016.132

16. Dai D, Wang H, Zhu L, et al. N6-methyladenosine links RNA metabolism to cancer progression. Cell Death Dis. 2018;9(2):124. doi:10.1038/s41419-017-0129-x

17. Yang X, Shao F, Guo D, et al. WNT/ $\beta$-catenin-suppressed FTO expression increases m6A of c-Myc mRNA to promote tumor cell glycolysis and tumorigenesis. Cell Death Dis. 2021;12(5):462. doi:10.1038/s41419-021-03739-z

18. Shi Y, Fan S, Wu M, et al. YTHDF1 links hypoxia adaptation and non-small cell lung cancer progression. Nat Commun. 2019;10 (1):4892. doi:10.1038/s41467-019-12801-6

19. Lou X, Ning J, Liu W, et al. YTHDF1 promotes cyclin B1 translation through m6A modulation and contributes to the poor prognosis of lung adenocarcinoma with KRAS/TP53 co-mutation. Cells. 2021;10 (7):1669. doi:10.3390/cells10071669

20. Chen Y, Lin Y, Shu Y, et al. Interaction between N6-methyladenosine (m6A) modification and noncoding RNAs in cancer. Mol Cancer. 2020;19(1):94. doi:10.1186/s12943-020-01207-4

21. Ma JZ, Yang F, Zhou CC, et al. METTL14 suppresses the metastatic potential of hepatocellular carcinoma by modulating $\mathrm{N}^{6}$ methyladenosine-dependent primary microRNA processing. Hepatology. 2017;65(2):529-543. doi:10.1002/hep.28885

22. Meyer KD, Jaffrey SR. Rethinking m6A readers, writers, and erasers. Annu Rev Cell Dev Biol. 2017;33:319-342. doi:10.1146/annurevcellbio-100616-060758

23. Zaccara S, Ries RJ, Jaffrey SR. Reading, writing and erasing mRNA methylation. Nat Rev Mol Cell Biol. 2019;20(10):608-624. doi:10.1038/s41580-019-0168-5

24. Shi H, Wei J, He C. Where, when, and how: context-dependent functions of RNA methylation writers, readers, and erasers. $\mathrm{Mol}$ Cell. 2019;74(4):640-650. doi:10.1016/j.molcel.2019.04.025

25. Deng S, Zhang H, Zhu K, et al. M6A2Target: a comprehensive database for targets of m6A writers, erasers and readers. Brief Bioinform. 2021;22(3):bbaa055. doi:10.1093/bib/bbaa055

26. Chen W, Zheng R, Baade PD, et al. Cancer statistics in China, 2015. CA Cancer J Clin. 2016;66(2):115-132. doi:10.3322/caac.21338

27. Yang Y, Ding L, Hu Q, et al. MicroRNA-218 functions as a tumor suppressor in lung cancer by targeting IL-6/STAT3 and negatively correlates with poor prognosis. Mol Cancer. 2017;16(1):141. doi:10.1186/s12943-017-0710-z

28. Travis WD. Update on small cell carcinoma and its differentiation from squamous cell carcinoma and other non-small cell carcinomas. Mod Pathol. 2012;25:S18-S30. doi:10.1038/modpathol.2011.150 
29. Mao Y, Xue P, Li L, et al. Bioinformatics analysis of mRNA and miRNA microarray to identify the key miRNA-gene pairs in small-cell lung cancer. Mol Med Rep. 2019;20(3):2199-2208. doi:10.3892/mmr.2019.10441

30. Cao Z, Pan X, Yang Y, et al. The lncLocator: a subcellular localization predictor for long non-coding RNAs based on a stacked ensemble classifier. Bioinformatics. 2018;34(13):2185-2194. doi:10.1093/ bioinformatics/bty085

31. Huang P, Xi J, Liu S. MiR-139-3p induces cell apoptosis and inhibits metastasis of cervical cancer by targeting NOB1. Biomed Pharmacother. 2016;83:850-856. doi:10.1016/j.biopha.2016.07.050

32. Zou ZC, Dai M, Huang ZY, et al. MicroRNA-139-3p suppresses tumor growth and metastasis in hepatocellular carcinoma by repressing ANXA2R. Oncol Res. 2018;26(9):1391-1399. doi:10.3727/ 096504018X15178798885361

33. Shi L, Yuan Y, Li HY. MicroRNA-139-3p suppresses growth and metastasis of glioblastoma via inhibition of NIN1/RPNI2 binding protein 1 homolog. Eur Rev Med Pharmacol Sci. 2019;23 (10):4264-4274. doi:10.26355/eurrev_201905_17931

34. Xue F, Li QR, Xu YH, et al. MicroRNA-139-3p inhibits the growth and metastasis of ovarian cancer by inhibiting ELAVL1. Oncol Rep. 2019;12:8935-8945. doi:10.2147/OTT.S210739
35. Zhang W, Xu J, Wang K, et al. miR-139-3p suppresses the invasion and migration properties of breast cancer cells by targeting RAB1A. Oncol Rep. 2019;42(5):1699-1708. doi:10.3892/or.2019.7297

36. Ma Y, Chen Z, Yu G. microRNA-139-3p inhibits malignant behaviors of laryngeal cancer cells via the KDM5B/SOX2 axis and the Wnt/ $\beta$ Catenin pathway. Cancer Manag Res. 2020;12:9197-9209. doi:10.2147/CMAR.S268871

37. Ni ZZ, He JK, Tang X, et al. Identification of ELAVL1 gene and miRNA-139-3p involved in the aggressiveness of NSCLC. Eur Rev Med Pharmacol Sci. 2020;24(18):9453-9464. doi:10.26355/ eurrev_202009_23030

38. Zhu Y, Zhou C, He Q. High miR-139-3p expression predicts a better prognosis for hepatocellular carcinoma: a pooled analysis. J Int Med Res. 2019;47(1):383-390. doi:10.1177/0300060518802727

39. Li Y, Ma C, Qian M, et al. Downregulation of NOB1 suppresses the proliferation and tumor growth of non-small cell lung cancer in vitro and in vivo. Oncol Rep. 2020;43(3):1031. doi:10.3892/ or.2020.7483

40. Liu K, Chen HL, Gu MM, et al. NOB1 expression predicts early response to cisplatin-based chemotherapy in patients with advanced non-small cell lung cancer. $J$ Chemother. 2016;28(3):225-229. doi:10.1179/1973947815Y.0000000041

\section{Publish your work in this journal}

Cancer Management and Research is an international, peer-reviewed open access journal focusing on cancer research and the optimal use of preventative and integrated treatment interventions to achieve improved outcomes, enhanced survival and quality of life for the cancer patient.
The manuscript management system is completely online and includes a very quick and fair peer-review system, which is all easy to use. Visit http://www.dovepress.com/testimonials.php to read real quotes from published authors. 